\title{
MÉTODOS DE INTERPOLAÇÃO PARA ESTIMAR O pH EM SOLO SOB DOIS MANEJOS DE CAFÉ ARÁBICA ${ }^{1}$
}

\author{
METHODS OF INTERPOLATION FOR ESTIMATING THE $p H$ IN SOIL \\ UNDER TWO MANAGEMENT OF COFFEE ARABIC
}

\author{
Alessandra Fagioli da Silva²; Julião Soares de Souza Lima³ ${ }^{3}$ Rone Batista de Oliveira ${ }^{4}$
}

\section{RESUMO}

Muitos são os métodos utilizados para estimar valores em locais não amostrados para construção de mapas de isolinhas. O objetivo deste estudo foi utilizar os métodos de interpolação krigagem, inverso do quadrado da distância e polinomial na representação da variabilidade espacial do $\mathrm{pH}$ do solo no manejo orgânico e convencional na cultura do cafeeiro. Para isso, foram construídas malhas irregulares para amostragem de solo na profundidade de $0-0,10 \mathrm{~m}$, totalizando 40 pontos amostrais em cada área. Para aferição dos métodos de interpolação foram retirados $10 \%$ do total de pontos, para cada área. Inicialmente, os dados foram avaliados através da estatística clássica (descritiva e exploratória) e análise espacial. O método inverso do quadrado da distância e a krigagem apresentam menor erro na estimativa dos dados. O método da krigagem apresentou menor variação em torno da média nos diferentes manejos.

Palavras chave: Geoestatística, estimativa, sistemas de manejo.

\section{ABSTRACT}

There are many methods used to estimate values in places no sampled for construction of contours maps. The aim of this study was to use the methods of interpolation kriging, inverse of the square of the distance and polynomial in the representation of the spatial variability of the $\mathrm{pH}$ of the soil in the organic and conventional management in the culture of the coffee plantation. For that, irregular meshes were built for soil sampling in the depth of 0-0,10 meters, totaling 40 points sampling in each area. For gauging of the interpolation methods they were solitary $10 \%$ of the total of points, for each area. Initially, the data were appraised through the classic statistics (descriptive and exploratory) and spatial analysis. The method inverse square of the distance and kriging has low error in estimating dados. The method of kriging presented low variation around the average in different managements.

Key words: Geostatistics, estimating, management systems.

\section{INTRODUÇÃO}

Muitos são os métodos utilizados para estimar valores em locais não amostrados. Para caracterizar bem uma determinada área, às vezes é preciso coletar um grande número de dados, o que em alguns casos se torna inviável a sua realização. Existem diversos esquemas amostrais para serem considerados, dependendo das características da região e natureza do fenômeno a ser medido. Uma certa aleatoriedade na amostragem é preferível no intuído de minimizar a presença de determinada tendência na distribuição, o que em alguns casos pode levar a erros na estimativa. Segundo Lamparelli et al. (2001), tem-se que usar o bom senso ou até um estudo prévio, para se fazer uma amostragem que contenha número suficiente de amostras, incluindo o espaçamento entre elas, a fim de que se consiga detectar alguma estrutura de correlação entre as amostras, e obter assim uma boa estimativa do modelo para explicar o padrão de variabilidade de cada parâmetro ou atributo considerado.

\footnotetext{
Artigo apresentado no XXXI Congresso Brasileiro de Ciências do Solo em Gramado-RS;

2 Graduando em agronomia, Centro de Ciências agrárias, Universidade Federal do Espírito Santo (CCA-UFES). Rua José Vieira Tatagiba, 61, Guararema, Alegre, ES, CEP: 29.500-000. Brasil. E-mail: alefagiolli@ yahoo.com.br

3 Eng. Agrícola, Professor Adjunto do Departamento de Engenharia Rural, CCA-UFES. Alto Universitário, s/n, Alegre, ES, CEP: 29.500-000. Brasil. E-mail: limajss@yahoo.com.br

$4 \quad$ Eng $^{\circ}$ Agrônomo, Doutorando na Universidade Estadual de São Paulo, UNESP, Botucatu, SP. Brasil E-mail: roneantiversus@ yahoo.com.br
} 
Na estatística clássica, que se baseia na distribuição normal, cada medida em um ponto de amostragem é tomada como uma variável aleatória $x_{i}$ independentemente das demais $x_{j}$, que totalizam $n$ medidas, na esperança de que os valores amostrados estejam em torno da média dos dados (Klaus \& Timm, 2004). Já na técnica das variáveis regionalizadas emprega-se uma amostragem na qual as coordenadas dos locais amostrados são de importância na análise estatística para a confecção de mapas, quando da presença da dependência espacial.

Para Fuks (1998) a caracterização dos parâmetros como responsáveis pelo sucesso da produção devem ser colocadas à disposição do usuário na forma de mapas. Segundo Burrough (1986), o procedimento de estimar o valor de um atributo em pontos não amostrados, através de valores conhecidos é denominado de interpolação.

Na cultura do café, conhecer e controlar a acidez do solo é fundamental, pois tanto a acidez do solo como a alcalinidade influencia na disponibilidade dos nutrientes necessários para as plantas. Normalmente, o solo ácido tem alta concentração de hidrogênio e alumínio, que são tóxicos para as plantas. Para o café, a faixa ideal de acidez ativa $(\mathrm{pH})$ está entre 5,5 e 6,5 (Dadalto \& Fullin, 2001).

Este trabalho teve como objetivo utilizar métodos de interpolação na representação da variabilidade espacial do $\mathrm{pH}$ do solo em dois manejos na cultura do cafeeiro.

\section{MATERIAL E MÉTODOS}

Os dados foram coletados em duas áreas comerciais de café arábica (Coffea arabica cv. catuaí 44) sob dois tipos de manejo do solo: orgânico (MORG) e convencional (MCON), no município de Irupi, região do entorno do Caparaó, sul do Estado do Espírito Santo. A região situa-se na Latitude de $20^{\circ} 20^{\prime} 43^{\prime \prime} \mathrm{S}$ e Longitude $41^{\circ} 38^{\prime}$ $28^{\prime \prime} \mathrm{W}$ do meridiano de Greenwich, com altitude de $765 \mathrm{~m}$ e temperatura média anual de $20^{\circ} \mathrm{C}$. O solo das lavouras cafeeiras é um Latossolo VermelhoAmarelo distrófico (EMBRAPA, 1999).

As lavouras de café têm aproximadamente dez anos de implantação. A primeira lavoura (MORG) após dois anos de implantação iniciou-se o processo de transição para orgânico e há três anos possui certificação nacional e há seis meses o selo internacional, constituindo um sistema orgânico consolidado. A segunda lavoura vem sendo conduzida por meio do sistema convencional de produção de café, ou seja, sistema comumente utilizado pelas propriedades cafeeiras, como adubação mineral e aplicação de herbicidas e capinas manual.

Na lavoura sob manejo orgânico (MORG), com espaçamento de $2 \times 1$ metros e o solo de textura média, uma vez por ano é aplicado $8 \mathrm{~kg}$ de composto orgânico por cova, produzido na própria propriedade. As plantas espontâneas nas entre linhas são controladas por roçadora motorizada costal. No período de chuvas é aplicado, mensalmente, na forma de pulverização foliar o humato de macota, produto de $\mathrm{pH}$ neutro, na proporção de $700 \mathrm{ml}$ do produto por 20 litros de água, utilizando no total de 200 a $220 \mathrm{~L} \mathrm{ha}^{-1}$.

A lavoura de manejo convencional (MCON) tem espaçamento de 3,0x1,5 metros. As plantas espontâneas que ocorrem nas entre linhas são controladas com o uso do herbicida Roundup uma vez por ano e por meio de capinas manuais, mantendo-se baixa cobertura no solo. Quanto à adubação é utilizado o formulado 20-05-20 (NPK) para nitrogênio $(\mathrm{N})$, fósforo $(\mathrm{P})$ e potássio $(\mathrm{K})$.

$\mathrm{O}$ manejo nutricional do café, nas duas áreas, como calagem e adubação do solo e foliar, é realizado através de aplicações uniformes em toda área, por meio de recomendação de dose única.

O esquema adotado para retiradas de amostras no experimento foi através do sistema de amostragem aleatória, conforme Lamparelli et al. (2001), com os pontos georreferenciados por um GPS, modelo GTR-1. As amostras de solo foram retiradas na profundidade de $0-0,10 \mathrm{~m}$ na projeção da copa do cafeeiro, na parte superior do declive. A determinação do $\mathrm{pH}$ em água foi segundo a metodologia da EMBRAPA (1997).

Para aferição dos métodos de interpolação foram retirados 4 pontos ( $10 \%$ do total) em cada área, com valores conhecidos e suas respectivas coordenadas. Para a comparação entre os métodos de interpolação determinaram-se o erro do valor estimado em relação ao medido, o erro médio e desvio padrão da média dos erros, conforme Weirich Neto et al. (2006).

Para obtenção dos mapas, utilizou-se de três métodos para estimativa do $\mathrm{pH}$, como: a krigagem (KRI), que é um método geoestatístico, que se utiliza um estimador linear não-viciado com mínima variância e leva em consideração a estrutura de 
variabilidade encontrada para o atributo, com a semivariância calculada pela equação abaixo:

$$
\gamma^{*}(h)=\left(\frac{1}{2 N(h)}\right) \sum_{i=1}^{N(h)}\left[Z\left(x_{i}\right)-Z\left(x_{1}+h\right)\right]^{2}
$$

em que: $\gamma^{*}$ a semivariância experimental, obtida pelos valores amostrados $\mathrm{Z}(\mathrm{xi}), \mathrm{Z}(\mathrm{xi}+\mathrm{h})$; h é a distância entre pontos amostrais; e N(h) o número total de pares.

A Equação 2 define o estimador de krigagem:

$$
\begin{gathered}
Z^{*}\left(x_{i}, x_{i}+h\right)=\sum_{i=1}^{n} \lambda_{i} Z\left(x_{i}, x_{i}+h\right) \\
\text { sujeito a } \sum_{i=1}^{n} \lambda_{i}=1
\end{gathered}
$$

em que $Z^{*}\left(x_{i}, x_{i}+h\right)$ é o estimador para um ponto $\left(x_{i}, x_{i}+h\right)$ da região e $\lambda_{i}$ são os pesos usados na estimação.

Outro método utilizado foi o do inverso do quadrado da distância (IQD), dado pela seguinte equação descrita por Mello et al. (2003).

$$
x_{p}=\frac{\sum_{i=1}^{n}\left(\frac{1}{d_{i}^{2}} * x i\right)}{\sum_{i=1}^{n}\left(\frac{1}{d_{i}^{2}}\right)}
$$

em que: $x_{p}=$ atributo interpolado; $x_{i}=$ valor do atributo do i-ésimo ponto de amostragem; $d_{i}=$ distância euclidiana entre o i-ésimo ponto de vizinhança e o ponto amostrado; e $\mathrm{n}=$ número de amostras.

A interpolação nestes dois métodos foi realizada no software $\mathrm{GS}^{+}$(Robertson, 2000).

A regressão múltipla, denominada de superficie de tendência, também, foi utilizada parar estimar os valores subtraídos da grade amostral. Este método baseia-se no ajuste de uma superfície contínua, segundo regressão por mínimos quadrados. A equação polinomial adotada foi a seguinte:

$$
\hat{y}=a_{0}+a_{1} x+a_{2} y+a_{3} x^{2}+a_{4} y^{2}+a_{5} x y
$$

em que: $\hat{y}$ é o valor estimado, $\mathrm{a}_{\mathrm{i}}$ são os coeficientes estimadores do modelo e $\mathrm{x}$ e $\mathrm{y}$ as coordenadas locais dos pontos amostrais.

Cabe ressaltar que existem outros métodos, bem como variações dentro de cada um, sendo que esses foram escolhidos por serem os mais aplicados.

Primeiramente, os dados passaram por uma análise descritiva para verificar a distribuição de freqüência, as medidas de posição e variação, a normalidade dos dados pelo teste Shapiro-Wilks a $5 \%$ de probabilidade e a presença de pontos discrepantes (outliers). Na análise geoestatística, no software $\mathrm{GS}^{+}$, utilizou-se para a escolha do modelo de ajuste da semivariância experimental os parâmetros soma dos quadrados dos resíduos; o coeficiente de determinação múltipla $\left(\mathrm{R}^{2}\right)$; e o índice de dependência espacial (IDE).

\section{RESULTADOS E DISCUSSÃO}

A Tabela 1 mostra os resultados da análise

\begin{tabular}{|c|c|c|c|c|c|c|c|c|c|c|c|c|}
\hline \multirow{2}{*}{ Manejos } & \multirow{2}{*}{$\mathbf{n}$} & \multirow{2}{*}{ Média } & \multirow{2}{*}{$\mathbf{s}$} & \multirow{2}{*}{ Md } & \multirow{2}{*}{ Min } & \multirow{2}{*}{ Max } & LI & $\mathbf{L S}$ & \multicolumn{3}{|c|}{ Coeficientes } & \multirow{2}{*}{$\begin{array}{c}\text { S-W } \\
\text { p-valor }\end{array}$} \\
\hline & & & & & & & \multicolumn{2}{|c|}{$(95 \%)$} & $\mathrm{CV}$ & Cs & Ck & \\
\hline MORG & 40 & 6,67 & 0,72 & 6,90 & 5,30 & 7,80 & 6,44 & 6,90 & 10,80 & $-0,53$ & $-0,83$ & 0,017 \\
\hline MCON & 40 & 4,88 & 0,44 & 4,80 & 4,20 & 5,80 & 4,74 & 5,00 & 9,03 & 0,37 & $-0,67$ & $0,051^{\mathrm{ns}}$ \\
\hline
\end{tabular}
descritiva e testes de normalidade do $\mathrm{pH}$ do solo

Tabela 1

Estatística descritiva do pH em solo cultivado com café arábica sob manejo orgânico (MORG) e convencional (MCON)

n: número de pontos amostrados; s: desvio-padrão; Md - mediana; Min- valor mínimo; Max: valor máximo; LI e LS: limite inferior e superior em torno da média a 95\%; CV: coeficiente de variação; Cs: coeficiente de assimetria; Ck: coeficiente de curtose; e p-valor: Teste de normalidade pelo Shapiro-Wilks a 5\% de probabilidade.

ns distribuição normal. 
na profundidade de 0-0,10 $\mathrm{m}$ em duas lavouras de café arábica, no manejo orgânico (MORG) e convencional (MCON).

Nota-se que o pH apresentou distribuição normal, pelo teste Shapiro-Wilks (S-W) a 5\% de probabilidade, somente no MCON. A assimetria negativa ocorreu no MORG, com a mediana maior que a média dos dados. $\mathrm{O}$ valor médio do $\mathrm{pH}$ no MCON indica solo ácido $(\mathrm{pH}<5,0)$, conforme Dadalto e Fullin (2001). Segundo Oliveira (2007), a acidificação do solo na cultura do café pode ocorrer pela aplicação de fertilizantes químicos e que uma calagem mais eficiente, mesmo que superficial, melhoraria a situação do solo e das plantas.

Os coeficientes de variação (CV) apresentaramse baixos $(<12 \%)$, segundo critérios propostos por Warrick \& Nielsen (1980). Segundo Davis et al. (1995), valor baixo de CV para o pH é devido ser este uma função logarítmica e, assim, apresenta naturalmente pequena variação.

A análise descritiva espacial, com os parâmetros de ajuste dos modelos teóricos ao semivariograma experimental, está apresentada na Tabela 2.

Na Figura 1, estão os semivariogramas experimentais escalonados pela variância dos dados, com amostras coletadas de 0-0,10 m de profundidade nas lavouras de café arábica em MORG e MCON.

O modelo esférico ajustou-se ao semivariograma experimental do $\mathrm{pH}$ nos dois manejos, porém apresentando moderada e forte dependência espacial para o MORG e MCON, respectivamente. Uma maior continuidade espacial foi detectado no MORG com alcance de 31,0 m, indicando que amostragens de solo devem ser realizadas à distância maior que no MCON, para que as amostras sejam consideradas independentes.

Os resultados dos métodos de interpolação por KRI, IQD e POL estão apresentados na Tabela 3. O MORG e MCON apresentam valores menores do erro médio $\left(\mathrm{e}_{\mathrm{M}}\right)$ para o IQD e KRI, respectivamente. A escolha do modelo teórico do semivariograma pode influenciar a KRI, bem como quando se considera o comportamento isotrópico do $\mathrm{pH}$ em todas às direções. Cabe ressaltar que, o software $\mathrm{GS}^{+}$utilizado adota a menor soma dos quadrados dos resíduos e o maior $\mathrm{R}^{2}$ na escolha do modelo de ajuste. Burrough (1986) não encontrou diferença nos valores interpolados pelos métodos IQD e KRI.

No MORG, o POL subestimou todos os valores reais e os superestimou no MCON, isto possivelmente
Tabela 2

Parâmetros dos semivariogramas ajustados para o atributo pH em solo cultivado com café arábica sob manejo orgânico (MORG) e convencional (MCON)

\begin{tabular}{|l|l|c|c|c|c|c|}
\hline Manejos & Modelo & $\mathbf{a}(\mathbf{m})$ & $\mathbf{C}_{\mathbf{0}}$ & $\mathbf{C}_{\mathbf{0}}+\mathbf{C}$ & $\begin{array}{c}\text { IDE } \\
(\boldsymbol{\%})\end{array}$ & $\begin{array}{c}\mathbf{R}^{\mathbf{2}} \\
(\boldsymbol{\%})\end{array}$ \\
\hline MORG & Esférico & 31,0 & 0,33 & 1,15 & 0,71 & 0,80 \\
\hline MCON & Esférico & 12,0 & 0,13 & 1,0 & 0,87 & 0,98 \\
\hline
\end{tabular}

a: alcance da dependência espacial; $\mathrm{C}_{0}$ : efeito pepita; $\mathrm{C}_{0}+\mathrm{C}$ : patamar; IDE: índice de dependência espacial e $\mathrm{R}^{2}$ : coeficiente de determinação múltipla.
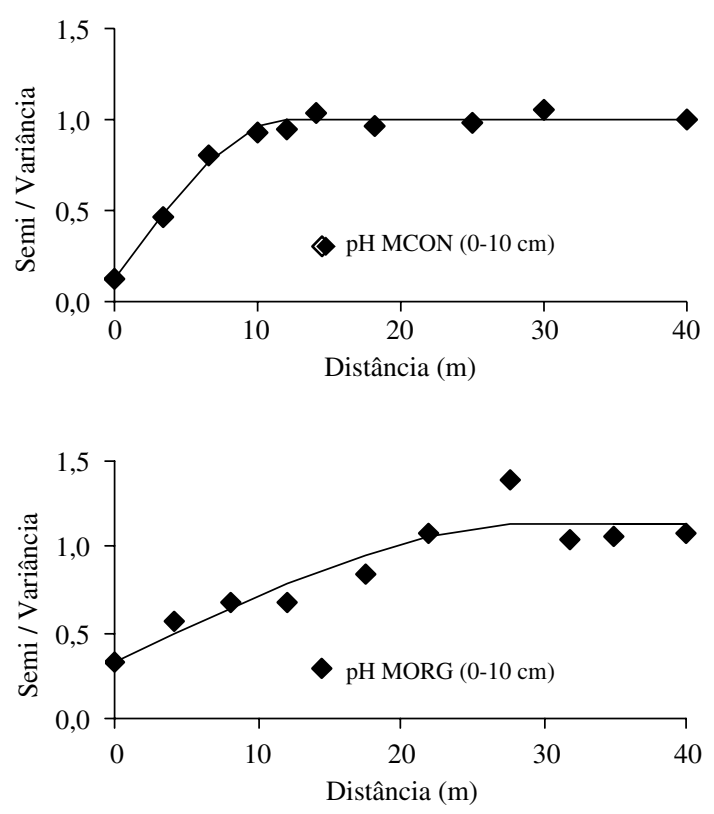

Figura 1. Semivariograma escalonado do $\mathrm{pH}$ no MORG e MCON.

ocorreu em virtude do número de amostras testes ser pequeno, o que pode ter influenciado no maior erro médio $\left(\mathrm{e}_{\mathrm{M}}\right)$ obtido nesse método.

Quanto ao desvio-padrão da média dos erros $\left(\mathrm{D}_{\mathrm{e}}\right)$, o método da krigagem apresentou menor variação em torno da média nos diferentes manejos.

No aspecto visual, no MORG e MCON os mapas temáticos para o $\mathrm{pH}$, apresentam uma certa similaridade quanto à forma entre os métodos IQD e KRI, provavelmente pela proximidade entre o desvio-padrão da média dos erros. 
Tabela 3

Valores do pH do solo medidos e estimados para cada um dos pontos a serem interpolados sob dois manejos: orgânico e convencional

\begin{tabular}{|c|c|c|c|c|c|c|c|c|c|c|c|c|c|}
\hline \multirow{2}{*}{ Valores } & & \multicolumn{6}{|c|}{ Manejo Orgânico } & \multicolumn{6}{|c|}{ Manejo Convencional } \\
\hline & & 1 & 2 & 3 & 4 & $\mathbf{e}_{\mathbf{M}}$ & $\mathbf{D}_{\mathrm{e}}$ & 1 & 2 & 3 & 4 & $\mathbf{e}_{M}$ & $\mathbf{D}_{\mathrm{e}}$ \\
\hline Real & & 6,80 & 7,50 & 7,00 & 5,30 & - & - & 5,20 & 4,60 & 4,90 & 5,70 & - & - \\
\hline \multirow{2}{*}{ KRI } & estimado & 7,22 & 6,70 & 6,20 & 6,14 & \multirow{2}{*}{11,03} & \multirow{2}{*}{1,51} & 4,89 & 4,74 & 4,86 & 4,95 & \multirow{2}{*}{5,74} & \multirow{2}{*}{1,99} \\
\hline & $\mathrm{E}(\%)$ & 6,18 & 10,67 & 11,43 & 15,85 & & & 5,96 & 3,04 & 8,82 & 13,16 & & \\
\hline \multirow{2}{*}{ IQD } & estimado & 7,11 & 6,7 & 6,63 & 6,19 & \multirow{2}{*}{9,33} & \multirow{2}{*}{2,54} & 4,79 & 4,70 & 4,84 & 4,85 & \multirow{2}{*}{7,55} & \multirow{2}{*}{2,23} \\
\hline & $\mathrm{E}(\%)$ & 4,56 & 10,67 & 5,29 & 16,79 & & & 7,88 & 2,17 & 5,22 & 14,91 & & \\
\hline \multirow{2}{*}{ POL } & estimado & 6,28 & 5,97 & 6,03 & 5,88 & \multirow{2}{*}{13,21} & \multirow{2}{*}{5,42} & 7,09 & 6,98 & 6,48 & 6,00 & \multirow{2}{*}{31,39} & \multirow{2}{*}{19,23} \\
\hline & $\mathrm{E}(\%)$ & 7,65 & 20,4 & 13,86 & 10,94 & & & 36,35 & 51,74 & 32,24 & 5,26 & & \\
\hline
\end{tabular}

e (\%): erro da estimativa; $\mathrm{e}_{\mathrm{M}}$ : erro médio da estimativa; $\mathrm{D}_{\mathrm{e}}$ : desvio-padrão da média dos erros; KRI: krigagem ordinária; IQD: inverso do quadrado da distância e POL: regressão polinomial.
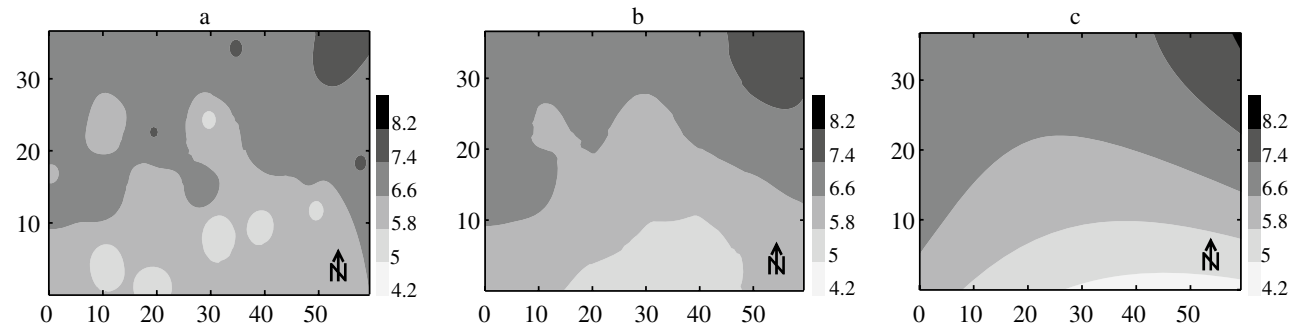

Figura 2. Mapa da interpolação pelo Inverso Quadrado da Distância (a), Krigagem (b) e Regressão Polinomial (c) para o manejo orgânico (MORG).

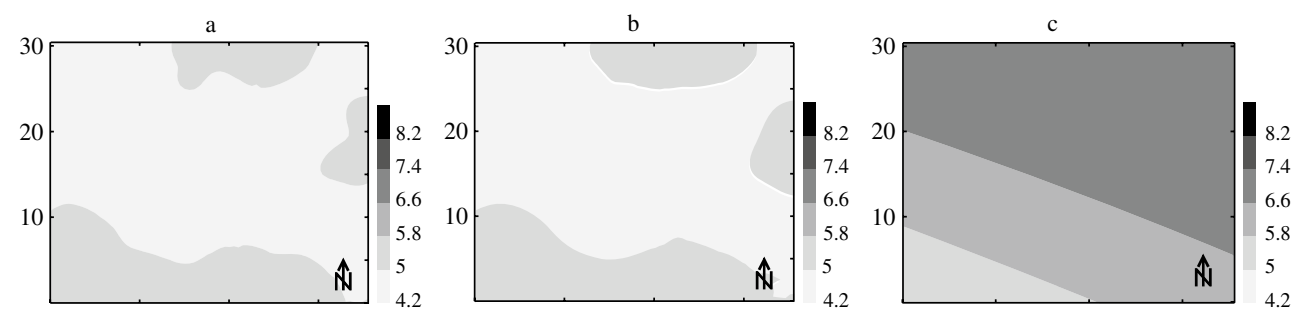

Figura 3. Mapa da interpolação pelo Inverso Quadrado da Distância (a), Krigagem (b) e Regressão Polinomial (c) para o manejo convencional (MCON).

\section{CONCLUSÕES}

- O método inverso do quadrado da distância e a krigagem apresentam menor erro na estimativa dos dados; e
- A krigagem apresenta menor desvio-padrão da média dos erros. 


\section{LITERATURA CITADA}

BURROUGH, P.A. 1986. Principles of geographical information Systems for Land Resources Assessment. New York: Oxford University Press, 193 p.

DADALTO, G.G.; FULLIN, E.A. 2001. Manual de Recomendação de Calagem e adubação para o estado do Espírito Santo: $4^{\circ}$ aproximação. Vitória, ES: SEEA/INCAPER, 266 p.

DAVIS, J.G.; HOSSNER, L.R.; WILDING, L.P.; MANU, A. 1995. Variability of soil chemical properties in two sandy dunal soils of niger. Soil Sci. v. 159, p. 321-330

EMBRAPA. 1997. Centro nacional de Pesquisa de Solos. Manual de métodos de análise de solo. 2. ed. Rio de Janeiro, 212 p.

EMPRESA BRASILEIRA DE PESQUISA AGROPECUÁRIAEMBRAPA. 1999. Centro Nacional de Pesquisa de Solos. Sistema Brasileiro de Classificação de Solos. Brasília, $412 \mathrm{p}$.

FUKS, S.D. 1998. Novos modelos para mapas derivados de informações de solos. In: ASSAD, E.D.; SANO, E.E. (Ed.) Sistemas de Informações Geográficas. 2. ed. Brasília: Serviço de Produção de Informação/Embrapa, cap. 19, p. 373-410.

KLAUS, R.; TIMM, L.C. 2004. Solo, planta e atmosfera: conceitos, processos e aplicações. Barueri - SP: Manole, 1 ed. 478 p.
LAMPARELLI, R.A.C; ROCHA, V.J.; BORGHI, E. 2001. Geoprocessamento e Agricultura de Precisão: fundamentos e aplicações. Guaíba: editora agropecuária Ltda, 118 p.

MELLO, C.R.; LIMA, J.M.; SILVA, A.M.; MELLO, J.M.; OLIVEIRA, M.S. 2003. Krigagem e Inverso do Quadrado da Distancia para Interpolação dos Parâmentros da Equação de Chuvas Intensas. R. Bras. Ci. Solo. V. 27, p. 925-933.

OLIVEIRA, R.B. 2007. de. Mapeamento e Correlação de Atributos do Solo e de Plantas de Café Conilon para fins de Agricultura de Precisão. 129f. Dissertação. Departamento de Engenharia Rural, Universidade Federal do Espírito Santo, Alegre.

ROBERTSON, G.P. 2000. GS ${ }^{+}$: Geostatistics for the environmental sciences GS+ User's Guide. Plainwell, Gamma Desing Software, $152 \mathrm{p}$.

WARRICK, A.W.; NIELSEN, D.R. 1980. Spatial variability of soil physical properties in the field. In: HILLEL, D (ed.). Applications of soil physics. New York : Academic, p. 319-344.

WEIRICH NETO, P. H; BUZOLINI JUNIOR, O.; ROCHA, J.V.; BORGHI, E.; SVERZUT, C.B. 2006. Variabilidae espacial do conteúdo de areia no solo, utilizando diferentes métodos de interpolação. R. Ci. Exatas Terra, Ci. Agr. Eng. Ponta Grossa, UEPG. v. 12, p. 41-49. 